\title{
Formación ciudadana: un reto para la convivencia
}

\author{
Ediliberto Hernández González ${ }^{*}$ \\ Johana Barreneche Corrales ** \\ Recibido: 25 de noviembre de 2016 \\ Enviado a pares evaluadores: 10 de diciembre de 2016 \\ Aprobado por pares evaluadores: 21 de enero de 2017 \\ Aprobado por comité editorial: 14 de febrero de 2017 \\ DOl: 10.22395/sye.v6n11a5
}

\section{RESUMEN}

El presente artículo documenta el proceso de evaluación y monitorización de un conjunto de actividades realizadas por la Universidad Católica de Manizales, en la ejecución del proyecto Formación Ciudadana: Un reto para la convivencia pacífica en el departamento de Caldas. Recoge, de manera sistemática, acciones y logros de cada una de las actividades desarrolladas con el objetivo de continuar fortaleciendo los procesos de formación en competencias ciudadanas.

Palabras clave: formación ciudadana, convivencia pacífica, municipio de Caldas (Colombia), comunidad educativa, Universidad de Manizales.

Doctor en Educación de la Universidad de la Salle, Magíste en educación de la Pontificia Universidad Javeriana y Licencido en filosofia de la Universidad Santo Tomas. Profesor e investigador de la Maestría en Educación en la Universidad Católica de Manizales. Correo electrónico: edilbertohernandez09@gmail.com

** Doctora de la Universidade Estadual de Campinas (Brasil), Magíster en Ciencia Política de la misma universidad y Comunicadora Social-Periodista de la Universidad del Valle. Profesora e investigadora de la Facultad de Comunicación de la Universidad de Medellín. Correo electrónico: jobarreneche@udem.edu.co 


\section{Civic formation: A challenge for coexistence}

\section{ABSTRACT}

This article documents the process of evaluation and monitoring of a set of activities carried out by the Universidad Católica de Manizales, in the implementation of the Citizen Training project: A challenge for peaceful coexistence in the estate of Caldas. It collects, in a systematic way, actions and achievements of each one of the activities developed with the objective of continuing to strengthen the training processes in citizen's competences.

Keywords: civic formation, peaceful coexistence, Caldas State (Colombia), educational community, University Catolic of Manizales. 


\section{Introducción}

El proyecto Formación ciudadana: un reto para la convivencia pacífica en el departamento de Caldas (2014) tuvo seis componentes y fue ejecutado atendiendo, entre otros, los siguientes objetivos: acompañar y asesorar a los establecimientos educativos en la institucionalización de las competencias ciudadanas; promover el desarrollo de competencias ciudadanas, comunicativas y emocionales en los docentes y demás integrantes de la comunidad; lograr la participación de la comunidad en espacios de diálogo, discusión y reflexión crítica frente al ejercicio de la ciudadanía, de los derechos humanos y de la sana convivencia; coordinar acciones dirigidas a la comunidad educativa entre los sectores e instituciones con responsabilidad en la formación ciudadana y la resolución pacífica de conflictos desde la convicción y la reflexión, y sistematizar el proceso formativo dando cuenta de la valoración de los indicadores propuestos en el proyecto, con fines de mejoramiento continuo (Puerta, 1996). La ejecución del proyecto se articuló a los procesos de investigación y proyección social de la Universidad Católica de Manizales, con el propósito de aunar esfuerzos y recursos que contribuyeran al desarrollo de cada actividad (Álvarez, 2007).

La forma en que se divide este documento, en seis partes, corresponde a la manera como las actividades dentro del proyecto fueron divididas y ejecutadas. La primera, referida a la asesoría y al acompañamiento para la institucionalización de las competencias ciudadanas en el Proyecto Educativo Institucional -PEIen los establecimientos educativos de los municipios de: Chinchiná, Villamaría, Palestina, Neira, La Dorada, Norcasia, Samaná, Victoria, Supía, Marmato, La Merced y Filadelfia. La asesoría estuvo centrada en el reconocimiento de las particularidades y los desarrollos realizados en cada localidad, con el propósito de fortalecer e incentivar los contextos y las prácticas en las que cada comunidad se encuentra comprometida.

La segunda comprende el desarrollo de tres cursos de actualización en competencias ciudadanas y resolución pacífica de conflictos, dirigidos a los docentes de los establecimientos educativos del departamento de Caldas con el propósito de promover la reflexión y la construcción de propuestas en torno a la convivencia ciudadana, a través del análisis de situaciones reales de la comunidad y el contexto; también se trabajó en torno a la participación y responsabilidad democrática mediante ejercicios puntuales que favorecieran la construcción de acuerdos sobre normas y decisiones en relación con la convivencia individual y colectiva.

La tercera parte corresponde a la realización de una serie de talleres de formación dirigidos a los niños y jóvenes integrantes de los comités estudiantiles y padres de familia de los municipios de Aguadas, Pácora, Salamina, Aranzazu, 
Anserma, Viterbo, Belalcázar, Risaralda, San José, Marquetalia, Manzanares, Pensilvania, Marulanda, Villamaría y Chinchiná.

La cuarta aborda la realización de trece (13) talleres teatrales realizados en los municipios de Aguadas, Pácora, Salamina, Aránzazu, Anserma, Viterbo, Belalcázar, Risaralda, San José, Marquetalia, Pensilvania, y Marulanda. Los talleres, en su mayoría, fueron desarrollados en las instituciones educativas normales superiores con el propósito de incentivar la réplica de la estrategia.

La quinta parte recoge las incidencias de los foros desarrollados en los municipios de Chinchiná y Riosucio. Los foros fueron entendidos como espacios de discusión e interacción comunitarios que posibilitaron poner el tema de la formación ciudadana y la resolución pacífica de los conflictos en la escena de las discusiones y las agendas de las organizaciones locales de cada municipio.

Finalmente, se reseñan los detalles de la elaboración de una Cartilla dirigida a educadores. Esta fue diseñada como una herramienta pedagógica para la formación en ciudadanía. La Cartilla contó con el debido sustento teórico y un lenguaje visual que recurre a elementos discursivos, estéticos y sensibles que van más allá de la lectura académica.

Es importante introducir también cómo se comprendieron, a lo largo del proyecto, las competencias ciudadanas y la ciudadanía. Las primeras se entendieron como el conjunto de conocimientos y habilidades que las personas, idealmente, deberían tener para actuar de manera constructiva en una sociedad democrática. Algunas de esas habilidades han sido agrupadas por tipos: 1. conocimientos, 2. habilidades cognitivas, 3. habilidades emocionales, 4. habilidades comunicativas, y 5. habilidades integradoras (E. Chaux, J. Lleras y A. Velásquez. 2012).

Durante el desarrollo del proyecto se les dio énfasis a las habilidades emocionales, comunicativas e integradoras. El trabajar con maestros de la red pública ofrecía dos presupuestos: primero, que ellos tendrían conocimientos básicos sobre asuntos relacionados con la democracia y el ejercicio ciudadano, es decir, que conocían los mecanismos existentes de participación, así como los derechos fundamentales, aspectos centrales de la formación ciudadana. Y segundo, porque las habilidades cognitivas, a saber: la toma de perspectiva, la interpretación de intenciones, la generación de opciones, la consideración de consecuencias, la meta-cognición y el pensamiento crítico se enmarcan dentro de las habilidades emocionales, comunicativas e integradoras.

Las habilidades emocionales son aquellas que permiten identificar y responder de manera adecuada a las emociones propias y a las de los otros. Las comunicativas son las que posibilitan dialogar con los otros argumentando sobre los propios puntos de vista, ideas, intereses y opiniones en general. Y las 
integradoras, siendo más amplias, articulan todas las habilidades anteriores para el manejo de diversas situaciones, entre ellas, la solución de los conflictos mediante el diálogo constructivo.

De otro lado, se entendió la ciudadanía partiendo de la idea de que la democracia se construye -y se preserva- culturalmente, lo que significa que debe existir una educación que facilite las condiciones para la manutención de la misma. En ese sentido, la ciudadanía a la que nos referimos es aquella que comprende que los individuos, seres auto-reflexivos por excelencia, tienen la posibilidad de decidir permanentemente sobre cada uno de sus actos; para ello, cabe a algunas instancias, ofrecer herramientas que contribuyan a la comprensión de dicha responsabilidad sobre la democracia.

\section{Asistencia técnica}

Este componente consistió en la realización de un taller de ocho (8) horas en doce (12) de los municipios de Caldas, con el propósito de asesorar y acompañar a los establecimientos educativos en las dinámicas de institucionalización de las competencias ciudadanas en el Proyecto Educativo Institucional. En este sentido, las actividades de cada uno de los talleres se orientaron a reconocer la importancia de la participación de todos los estamentos de la institución educativa en los procesos de integración de las competencias ciudadanas en los diferentes ambientes educativos (gestión institucional, instancias de participación, aulas de clases, proyectos pedagógicos y manejo del tiempo libre).

Teniendo en cuenta el número de horas definidos para cada taller, los recursos disponibles y la ubicación geográfica de los doce (12) municipios, se conformaron tres (3) zonas, asignando un profesional de la Universidad Católica de Manizales, a cada una de ellas.

\subsection{Perspectiva teórica y metodológica}

El taller que se desarrolló en cada uno de los doce (12) municipios tuvo como punto de partida el reconocimiento de las particularidades y desarrollos formativos realizados en el departamento de Caldas, en relación con las competencias ciudadanas; esto con el propósito de fortalecer los contextos y las prácticas con las que cada comunidad se encuentra comprometida, entendiendo que la formación en competencias ciudadanas no puede hacerse desde una lógica de quien sabe e impone un conocimiento, sino desde quien se acerca, reconoce y contribuye a potenciar los valores de cada comunidad (MEN, 2011). Por tanto, la formación en competencias ciudadanas, desde la perspectiva asumida por la Universidad Católica de Manizales, comprendió un proceso respetuoso de 
articulación de los saberes formales y teóricos, con los saberes ancestrales de convivencia que poseen las comunidades.

En tal sentido, se promovieron elementos en el nivel práctico y vivencial, necesarios en los particulares ambientes escolares donde convergen todos los miembros de la comunidad educativa. Es importante señalar que la institucionalización de las competencias ciudadanas en las instituciones educativas requiere que el trabajo se realice de forma transversal, coordinada y armónica en los ambientes de aprendizaje (MEN, 2006).

El encuentro particular con los docentes, directivos y en algunos casos con psico-orientadores -miembros de las comunidades educativas, delegados por las instituciones de acuerdo con las directrices de la Secretaría de Educación departamental- se realizó bajo la modalidad de talleres de asesoría y acompañamiento, donde se privilegió un ejercicio de reflexión en torno a la manera como en los establecimientos se están aprovechando los ambientes escolares para la formación ciudadana. La sesión se acompañó de aportes conceptuales y teóricos por parte de los profesionales responsables de la ejecución del taller, lo que facilitó la comprensión y apropiación en torno al sentido de las competencias ciudadanas; dichos aspectos se abordaron desde las orientaciones de la Cartilla n. ${ }^{\circ}$. (MEN 2011).

En un segundo momento, y asumiendo como base la Cartilla n. 2 (MEN 2011), se realizó la valoración del grado de institucionalización de las competencias ciudadanas; además, se tuvieron en cuenta los indicadores de proceso de mejoramiento, lo que implicó reflexiones en torno a los cuatro (4) estadios que sirven como mapa para caracterizar un momento de desarrollo. Esta perspectiva facilitó la planeación y ejecución de acciones, estructura que está en sintonía con la organización por procesos y componentes desarrollados ampliamente por el Ministerio de Educación Nacional (MEN, 2008).

\subsection{Desarrollo de los talleres en los municipios}

El proceso de convocatoria para la participación en los talleres fue realizado por la SED a través de directores de núcleo y rectores de las instituciones educativas en cada municipio. En coordinación con los mismos, se determinó el lugar más propicio para la realización del encuentro. Las condiciones de una jornada de ocho (8) horas continuas implicaron la planeación de talleres con momentos claramente definidos y la incorporación de elementos lúdicos que favorecieran la construcción de aprendizajes y ayudaran a mantener el interés y la receptividad por parte de los participantes. En los talleres participaron un promedio de dieciséis (16) educadores por municipio; ciento noventa y nueve (199) en total. 
Es así como en un primer momento se realizó una actividad lúdica con el propósito de facilitar la presentación y animación de los directivos y docentes para el desarrollo del taller, además de dar lugar al reconocimiento de presaberes con respecto a las competencias ciudadanas. Para facilitar el compartir y posibilitar un primer nivel de reflexión, se les propuso, a cada participante, diligenciar un instrumento con preguntas alusivas al tema de las competencias.

El taller continuó con una sesión de presentación teórica del tema de las competencias ciudadanas a través de diapositivas, haciendo énfasis en las estrategias de integración al currículo, así como algunas sugerencias para vivenciarlas en diversos momentos de la vida institucional. Este fue un espacio dialógico interesante en el que se pudo apreciar que en su mayoría, los docentes promueven las competencias ciudadanas a través de actitudes y actividades cotidianas y que solo en casos puntuales, las competencias ciudadanas constituyen un capital estructural de los procesos formativos en las instituciones.

Posteriormente se propuso a los participantes del taller un trabajo en grupos. A cada uno se le presentó un desafío que comprendía la descripción de una situación escolar, y se les sugirió que la solución al mismo fuera ofrecida desde la perspectiva de las competencias ciudadanas.

Continuando con la dinámica del taller se realizó un segundo momento teórico, orientado a promover la reflexión en torno a las posibilidades que tienen los miembros de las comunidades educativas de articular las competencias ciudadanas a los cinco ambientes institucionales: gestión institucional, instancias de participación, aulas de clases, manejo del tiempo libre y proyectos pedagógicos.

Finalmente como cierre del taller se propuso a los participantes el desarrollo de una actividad en grupos pequeños. A cada uno se les ofreció una imagen recortada en forma de rompecabezas y se les pidió que de manera participativa reconstruyeran la imagen y que a partir de ella plantearan algunos de los compromisos en torno a la institucionalización de las competencias ciudadanas suscitadas por la jornada.

\subsection{Resonancias de la asistencia técnica}

Los profesionales que participaron como talleristas en el componente de asistencia técnica en los municipios establecidos dentro del proyecto: Formación ciudadana, un reto para la convivencia pacífica en el departamento de Caldas evidenciaron un ambiente de participación, confrontación de saberes y disposición en general por parte de los docentes, psico-orientadores y directivos anfitriones. Percibieron que las instituciones educativas poseen diferentes estrategias y herramientas para la institucionalización de las competencias ciudadanas, pero 
que se trata de acciones aisladas que no responden a un proceso planificado y, por tanto, no existe un proceso sistematizado que garantice una dinámica de mejoramiento continuo.

Es importante resaltar el taller realizado en el municipio de Palestina, el cual, por solicitud de algunos maestros de la localidad, se realizó en una de las instituciones del sector rural y donde el jefe de núcleo del sector estuvo presente buena parte de la jornada. En este taller los participantes mostraron amplios niveles de dominio conceptual respecto de las competencias ciudadanas, y evidenciaron también una disposición singular frente a las actividades propuestas. Mostraron abiertamente cómo se están institucionalizando las competencias y su contribución a la formación de mejores seres humanos.

Al terminar los encuentros, los docentes y docentes-directivos mostraron interés en comprometerse de manera más decidida a incorporar en la vida de sus comunidades educativas las competencias ciudadanas e iniciar procesos de institucionalización más estructurados, de forma que se puedan valorar los avances y consolidar dichas prácticas. En algunos casos puntuales se evidenció discordancia de algunos participantes respecto a sus múltiples ocupaciones, pues manifestaron que cada vez se les pide que realicen más actividades en los mismos tiempos. Se cuestionó el papel del Estado frente a los compromisos con los requerimientos que demanda a través de los diversos informes que se solicitan y de los cuales no se evidencian ejercicios de realimentación.

\section{Formación: cursos de actualización en competencias ciudadanas}

El segundo componente del proyecto Formación ciudadana: un reto para la convivencia pacífica en el departamento de Caldas consistía en la oferta de tres (3) cursos de actualización en competencias ciudadanas y resolución pacífica de conflictos para ciento setenta (170) docentes de los establecimientos educativos del departamento.

Se esperaba que los cursos se realizaran mediante talleres teórico-prácticos donde se trabajaran diversas estrategias pedagógico-didácticas que nutrieran las prácticas de aula en las instituciones, entre estas, trabajo con dilemas morales, manejo de la asertividad, resolución de conflictos y otras actividades que apuntaran al desarrollo de las competencias ciudadanas, en particular las emocionales, cognitivas y comunicativas y su relación e integración con las distintas áreas del conocimiento (Chaux \& otros, 2004).

En tal sentido, se consideró importante tener como fundamento la Ley 1620 del 15 de marzo de 2013 "Por la cual se crea el Sistema Nacional de Convivencia Escolar y formación para el ejercicio de los derechos humanos, la educación para 
la sexualidad y prevención y mitigación de la violencia escolar" y su Decreto Reglamentario 1965 de septiembre de 2013 (Chaux, Vargas, Ibarra \& Minsk, 2013).

Para la realización de los cursos, la SED (Secretaría de Educación departamental) realizó una convocatoria entre todos los directivos, psico-orientadores y educadores del departamento de Caldas. Por su parte, la Universidad Católica de Manizales planificó los cursos a partir de los siguientes objetivos: promover el desarrollo de competencias ciudadanas, comunicativas y emocionales en los docentes y demás integrantes de la comunidad; incentivar en los docentes la importancia de las competencias ciudadanas, comunicativas y emocionales para mejorar la convivencia en la comunidad a través de actividades de actualización; y valorar la importancia que tiene la sana convivencia y sus repercusiones en el mejoramiento de las relaciones interpersonales en la comunidad.

Entre las dos entidades se acordó que los tres cursos previstos en el proyecto se realizarían de manera simultánea con la intención de garantizar mayor número de asistentes a todos los talleres y mejor calidad en el desarrollo de los mismos, teniendo en cuenta las limitaciones presupuestales del proyecto. Pensando que estas se pueden constituir en oportunidades, se asumió una lógica de encuentro académico, alternando espacios de conferencias colectivas, invitando como orientadores de los mismos a profesores reconocidos en la ciudad de Manizales por sus cualidades humanas y desarrollos académicos pertinentes. Los talleres se realizaron en grupos más pequeños, para lo cual la Universidad Católica de Manizales facilitó la participación de algunos profesores de la Maestría en Educación.

\subsection{Perspectiva teórica y metodológica}

Los cursos de actualización en competencias ciudadanas promovieron la reflexión, el análisis y la construcción de propuestas en torno a la convivencia ciudadana. Se profundizó en el conocimiento de leyes y normas del país referidas a las competencias para la vida en comunidad, a los mecanismos de participación y responsabilidad en los procesos sociales, al respeto por la vida y el medio ambiente, a la democracia, a las políticas de género y a las situaciones cotidianas que circundan el contexto educativo y comunitario (MEN, 2010).

En el desarrollo de los cursos se privilegiaron algunas estrategias como el juego, la creatividad, la proximidad y la confianza. En todo momento se procuró que los maestros se sintieran confortables para la apertura y el diálogo, y que en medio de un clima afectivo propicio, el compartir fuera ameno y espontáneo.

Las habilidades que se deberían desarrollar idealmente para actuar de manera constructiva en una sociedad democrática en el contexto colombiano han 
sido agrupadas, desde el Ministerio de Educación Nacional, en conocimientos, habilidades cognitivas, habilidades emocionales, habilidades comunicativas y habilidades integradoras (Chaux \& otros, 2004)

Los cursos de actualización, promovidos por la Universidad Católica de Manizales, enfatizaron únicamente en las habilidades emocionales, comunicativas e integradoras. El trabajar con maestros del sector público del departamento de Caldas ofrecía como presupuesto que conocían los mecanismos existentes de participación, así como los derechos fundamentales, es decir, aspectos centrales de la formación ciudadana. De otro lado, las habilidades cognitivas, a saber: la ampliación de perspectiva, la interpretación de intenciones, la generación de opciones, la consideración de consecuencias, la metacognición y el pensamiento crítico se encuadran, de alguna manera, dentro de las habilidades emocionales, comunicativas e integradoras. Por ello, no se privilegiaron, durante los talleres, las habilidades catalogadas como conocimientos y habilidades cognitivas.

Las habilidades emocionales se comprenden como aquellas que permiten identificar y responder de manera adecuada a las emociones propias y a las de los otros. Las comunicativas, a su vez, son aquellas que nos posibilitan encontrarnos con los otros desde las posibilidades que nos ofrece la conversación, proponiendo nuestros puntos de vista, ideas, intereses y nuestra visión del mundo. Por su parte, las habilidades integradoras, siendo más amplias, articulan todas las habilidades anteriores para el manejo de diversas situaciones, entre ellas, la solución de los conflictos mediante la creatividad y el diálogo constructivo (Chaux \& otros, 2004).

\subsection{Desarrollo de los cursos}

Acorde con lo planeado entre la SED y la Universidad Católica de Manizales, los tres cursos contemplados en el proyecto se realizaron de manera simultánea. Participaron ciento treinta y cuatro (134) educadores de colegios y escuelas del departamento de Caldas.

El encuentro se inició con una sesión inaugural en las instalaciones del aula máxima de la Universidad Católica de Manizales -Auditorio Santo Domingo-. Seguidamente se realizó la conferencia: Ética Moral y Ciudadanía, a cargo del profesor Jaime Alberto Restrepo, psicólogo, magíster en Educación y doctor en Ciencias Sociales.

Su charla estuvo centrada en la necesidad de desarrollar capacidades para vivir con otros en los escenarios sociales. Con tal propósito compartió ideas y experiencias investigativas alrededor de la empatía, la capacidad de cuidar a los demás y de forjar ideales regulativos, elementos que son condición para construir 
calidad de vida. Concluyó que la construcción de la ciudadanía pasa por una reflexión ética, referida a pensar la felicidad, la vida buena, lo comunitario y el reconocimiento de las necesidades de los otros. De forma secuencial cada grupo tuvo la posibilidad de participar en los tres talleres previstos para la jornada, los cuales se reseñan brevemente:

Taller n. 1: Manejo de emociones. Orientado por el Mg. Diego Armando Jaramillo, profesor de la Maestría en Educación de la UCM. Se trabajó a partir de la comprensión de la importancia del movimiento del cuerpo y de algunas frases provocadoras como: "Es incapaz de experiencia aquel a quien nada le pasa, a quien nada le acontece, a quien nada le sucede, a quien nada le llega, a quien nada le afecta, a quien nada le amenaza, a quien nada le hiere." (Larrosa, Skliar. 2009). De esta manera se promovieron, en los participantes, múltiples reflexiones en torno de lo que significa el encuentro con el otro (Skliar, 2007).

Taller n. 2: Desarrollo de habilidades comunicativas: Orientado por Dra. Johana Barreneche Corrales, profesora de la Maestría en Educación de la UCM. La profesora partió de la idea de que nuestros cuerpos dicen a pesar nuestro, y aunque permanezcamos en silencio (nos), comunicamos; decimos no solo aquello que parte de nuestra voluntad, con el deseo de ser transmitido, porque todo aquello que somos o hacemos puede ser leído por los otros. En torno de esta y de otras reflexiones giraron algunas de las conversaciones propuestas durante los encuentros en este taller.

Taller n. ${ }^{\circ}$ 3: Manejo y resolución de conflictos: Orientado el Mg. Mauricio Orozco, profesor de la Maestría en Educación de la UCM. El profesor emplea en la actividad reflexiones de diversos autores, entre ellos Estanislao Zuleta, quien afirma que deseamos mal. En lugar de desear una relación humana inquietante, compleja y perdible, que estimule nuestra capacidad de luchar y nos obligue a cambiar, deseamos un idilio sin sombras y sin peligros, un nido de amor, y por lo tanto, en última instancia, un retorno al huevo. En vez de desear una sociedad en la que sea realizable y necesario trabajar arduamente para hacer efectivas nuestras posibilidades, deseamos un mundo de satisfacción, una monstruosa sala-cuna de abundancia pasivamente recibida (Elogio de la dificultad) ${ }^{13}$.

La jornada de cierre de los cursos se inició con la conferencia del filósofo, magíster en Filosofía y doctor en Ciencias Sociales, profesor Jaime Alberto Pineda Muñoz, quien puso en escena una charla, con carácter performático, intercalando imágenes, poesía y conceptualizaciones que entrelazan el saber con sus repercusiones en las emociones humanas. Hizo énfasis en la necesidad de formar la mirada para tiempos de paz, puesto que nuestra mirada se ha cons-

Palabras pronunciadas por Estanislao Zuleta cuando, en 1980, recibió el título de Doctor Honoris Causa en Psicología de la Universidad del Valle. 
tituido en tiempos de guerra y el horror se ha hecho, con demasiada frecuencia, compañero de viaje.

Posteriormente, algunos de los voceros de los grupos, constituidos el día anterior, presentaron sus perspectivas de la experiencia. Además de manifestar su satisfacción con la realización del encuentro, expresaron cómo esta experiencia se articuló a sus sentimientos humanos más profundos y los estimuló a ser, ante todo, mejores seres humanos y mejores educadores.

\section{Formación: talleres de juego de roles}

Este componente del proyecto: Formación Ciudadana: un reto para el Departamento de Caldas comprendía la realización de talleres dirigidos a niños, jóvenes integrantes de los comités estudiantiles y padres de familia en 15 municipios del departamento de Caldas, actividad que se realizó empleando la estrategia "juego de roles", herramienta que facilita "orientar la construcción de valores sociales -base de la convivencia ciudadana- como el respeto a la diferencia, a la libre elección y la participación en decisiones de interés general" MEN (2010).

El uso de esta estrategia educativa facilitó hacer visibles las problemáticas en torno a los conflictos más relevantes del contexto y vislumbrar las soluciones a partir de casos específicos, dilemas morales y reflexión crítica. La estrategia favoreció la participación de la comunidad estudiantil y de padres de familia, y las actividades estuvieron orientadas a generar reflexiones en torno a la formación ciudadana, a la resolución pacífica de los conflictos en la vida cotidiana, así como al fortalecimiento de la formación ciudadana para la construcción de comunidades en el ámbito escolar que fomenten la convivencia pacífica en sus municipios.

La ejecución de estos talleres estuvo precedida de una serie de reuniones entre el equipo gestor de la SED y el equipo de la Universidad Católica de Manizales. En estas reuniones se acordó, entre otras cosas, el calendario de ejecución y las instituciones educativas de cada municipio en las cuales se realizaría la actividad. Se estableció también que la SED sería la responsable de las convocatorias.

\subsection{Descripción de los talleres}

El proyecto contemplaba un número de setenta (70) participantes por taller; esto implicó la realización de dos sesiones, cada una con un tiempo de dos (2) horas.

La sesión se inició con un saludo y las presentaciones de los participantes y el facilitador encargado de orientar el taller. Seguidamente se realizó una 
actividad de reconocimiento del grupo a través de la dinámica "fuera de tu silla". Esta actividad creó un clima de confianza entre los participantes.

En un segundo momento se presentaron los objetivos y la metodología para el desarrollo del taller. De forma particular se invitó a los participantes para que expusieran las problemáticas que más les preocupaban de su institución educativa, su grupo de amigos y su municipio en general. Para esta actividad se le propuso al grupo el empleo de la técnica de la cartografía social, situando sobre un mapa de la institución, las diferentes problemáticas que identificaron.

En el trascurso de la actividad los participantes fueron orientados a reconocer que no todo conflicto es negativo, que estos pueden ser oportunidades de aprendizaje y posibilidades para la construcción de acuerdos que movilicen soluciones a largo plazo. También se les indicaron salidas comprensivas para dar manejo a las diferencias cuyo punto de partida es aprender a reconocerlas.

Como cierre de la actividad se propuso la participación espontánea a manera de lluvia de ideas donde los participantes expresaron sus emociones, sentimientos y reflexiones frente a las actividades, los conflictos que fueron identificados y los medios que pusieron en práctica para resolverlos.

En el encuentro con los padres de familia se realizó una actividad inicial de sensibilización; para ello se empleó la técnica del "cuchicheo", actividad que facilitó la participación y el interés sobre el tema. Para el desarrollo del taller se les pidió que escribieran en pequeñas hojas las situaciones que más les molestaban en el día a día, luego elaboraron un listado. Estas ideas fueron compartidas entre todos y en la medida que se iban escuchando, cada participante iba inflando un globo, como una metáfora de las dimensiones que pueden tomar los conflictos.

Durante el transcurso del taller se fue promoviendo el proceso de reflexión crítica frente a la manera como los seres humanos relacionan el mundo de lo afectivo con el conflicto y su validación por la vía de la comunicación. Se invitó igualmente a los participantes a detectar la manera como reaccionan frente a situaciones límites de sus vidas a partir de una serie de posibilidades que remiten a formas habituales de ser ante el conflicto.

\subsection{Reflexiones en torno al desarrollo de los talleres}

En términos generales el encuentro con las comunidades educativas fue gratificante. En muchas de ellas se percibió receptividad frente a las estrategias que apuntan a consolidar procesos de incorporación de las competencias ciudadanas y mejorar los climas de convivencia escolar. Se pudo observar el interés en aprovecharlas para nutrir las dinámicas escolares y asumirlas como referentes para dar sentido a otros escenarios. 
Los talleres fueron bien recibidos por los estudiantes y padres de familia. En casi todos los municipios se reconoció la importancia, pertinencia e interés de los mismos en relación con el mejoramiento de la vida escolar, familiar y social, manifestando que se debe construir una formación ciudadana con responsabilidad y respeto. Los participantes más jóvenes también se mostraron receptivos y motivados a realizar actividades novedosas en medio de su cotidianidad, compartieron sus propias reflexiones en cuanto a asumir posturas críticas, realización de comentarios positivos e identificación de sus problemáticas tanto en la convivencia escolar como en su vida familiar.

Los grupos de estudiantes con los cuales se realizaron las actividades, se caracterizaron por ser proactivos, creativos y motivados; valoraron los ejercicios de la cartografía social en el sentido de que a través de estos pudieron opinar, reflexionar y argumentar desde su cotidianidad escolar. Se pudo evidenciar que el interés de los grupos crecía a medida que se sentían escuchados y valorados como actores importes en los procesos de transformación de sus problemáticas y los conflictos propios de sus municipios.

Asimismo, la participación de los padres de familia fue excelente; se motivaron mucho con los ejercicios propuestos. La asistencia de estos no fue la esperada, situación que puede ser comprendida desde diferentes circunstancias y que sin embargo, no afectó el desarrollo de la actividad, dado que en cada municipio se realizó con la población que se presentó. En ocasiones el número de asistentes favoreció la escucha de sus experiencias y vivencias, lo que se capitalizó en función de los ejercicios propuestos.

Es de anotar que en algunas instituciones los directivos y docentes se limitaron a organizar a los estudiantes y se ausentaron prontamente del escenario del taller manifestando tener otras ocupaciones. Esta actitud evidenció, de algunas maneras, la dificultad de generar procesos de transformación a largo plazo, dado que las estrategias promovidas desde las instancias gubernamentales no son suficientes por sí mismas, y se requiere de los educadores locales para que haya repercusión de dichas estrategias.

\section{Movilización social: talleres teatrales}

Este componente comprendió la realización de trece (13) talleres teatrales de significación para la formación en ciudadanía y resolución pacífica de conflictos. La actividad estuvo estructurada en dos momentos: una jornada de ocho (8) horas de taller con un grupo de doce (12) estudiantes, y una jornada de cuatro (4) horas para desarrollar una puesta en escena con un grupo de personas de la comunidad educativa. 
Los talleres teatrales se llevaron a cabo bajo la estrategia: teatro foro, retomando para ello diversas prácticas realizadas en otros países donde el teatro es empleado como recurso para la reflexión en torno a la convivencia, tal como lo propone el dramaturgo, actor, director y pedagogo teatral brasileño Augusto Boal (1931-2009), "En el teatro foro, el espectactor sube al escenario y ensaya lo que podría ser posible hacer en la vida real. A veces la solución de los problemas de los espectactores dependen de ellos mismos, de su propio deseo individual, de sus esfuerzos..." (Boal, 2009).

La estrategia del teatro foro, desarrollada por Boal, fue adaptada a los fines educativos del proyecto, por lo que este adquirió las características de teatro foro para la convivencia, con el propósito de hacer visibles las problemáticas en torno a los conflictos más relevantes del entorno y promover soluciones concertadas a partir de casos, dilemas morales y reflexión crítica.

La realización de los talleres se planificó para los municipios de Aguadas, Pácora, Salamina, Aranzazu, Anserma, Viterbo, Belalcázar, Risaralda, San José, Marquetalia, Manzanares, Pensilvania y Marulanda. La ejecución de los talleres la planificó el equipo gestor de la SED y el equipo de la Universidad Católica de Manizales. Entre otras cosas, se acordó privilegiar a las instituciones educativas normales superiores con la pretensión de incentivar procesos y garantizar mayores niveles de apropiación y réplica de la estrategia. Con base en este criterio se definió el correspondiente cronograma. Se estableció también que la SED sería la responsable de la comunicación con la respectiva institución donde se realizaría la actividad.

\subsection{Descripción de los talleres}

Respondiendo a las expectativas de las instituciones, los talleres de teatro para la convivencia se realizaron con la participación de grupos entre trece (13) y diecisiete (17) participantes. Cabe decir que el teatro para la convivencia, apoyados en Boal, es una estrategia de reflexión sobre la convivencia y un ensayo para configurar acciones futuras. Se trata de una dinámica donde en el presente se vuelve a vivir el pasado, para diseñar el futuro.

La primera jornada se trabajó en torno a las siguientes temáticas: reconocimiento del grupo y del espacio, dinamización del grupo y desarrollo de confianza y una introducción básica al teatro clásico y la variante del teatro foro. Para el desarrollo de estos temas se realizaron actividades de calentamiento y entrenamiento de la mirada. Para ello se sugirió el cambio de lugares por contacto visual; círculo de nombres, para lo que se propuso decir el nombre con gestos y repetición en coro por parte del grupo; caminar en el espacio con diferentes velocidades, movimientos libres con música, presentación en parejas, círculo de palmadas y de vocales. 
Posteriormente se dio la creación y selección de propuestas temáticas, análisis temático a través del teatro imagen y consolidación de una escena de teatro foro, técnicas de ensayo para la puesta en escena y, finalmente, se configuró la puesta en escena.

En la jornada de la mañana se dedicó tiempo para realizar ajustes a las escenas seleccionadas y a los ensayos correspondientes. Seguidamente se hizo una introducción al público de la actividad de la cual iban a hacer parte. Se les compartió que en el teatro tradicional ellos serían los espectadores, pero que aquí serían espectactores, lo que significa que tendrían la tarea de interactuar permanentemente con los actores. Se informó que en primera instancia los actores presentarían de manera secuencial toda la escena tal como había sido construida.

En una segunda oportunidad la escena sería intervenida, según los intereses del público. Cada vez que alguien del público levantara la mano, los actores quedarían estáticos, y de acuerdo con sus indicaciones la escena sería transformada. El público indagaba por las razones de ciertas actitudes de padres, profesores, estudiantes, y le proponían a los actores que cambiaran de roles para explorar qué sucedería.

\subsection{Reflexiones y aportes del desarrollo de los talleres}

Los rectores y psico-orientadores con los cuales se tuvo la oportunidad de conversar consideraron que los talleres incidieron positivamente en la vida de los chicos que participaron de ellos; el compromiso adquirido a lo largo de la jornada hablaba por sí mismo. En varios de los municipios se resaltó la importancia de que los chicos con potencial artístico tuviesen la oportunidad de mostrar sus talentos.

En términos comunitarios se consideró que los talleres contribuyeron al fortalecimiento de vínculos afectivos y a la cohesión de los grupos y de las familias. Las escenas representadas generaron inquietudes respecto al manejo de las pautas de crianza en forma continua y negociada, y a la necesidad de fortalecer la comunicación, los valores y principios que movilizaron las acciones de los miembros de las familias, así como a la importancia de reconocer las historias de vida, lo que permite mayores oportunidades frente a la labor formadora de la familia y las instituciones educativas.

Como reflexiones suscitadas al final de los talleres se pudo resaltar, entre otros temas, el reconocimiento del papel que juega la familia en la formación y educación de sus hijos; y el compromiso de los padres y madres llamados a fortalecer valores y principios de vida, lo que se constituye en clave para mini- 
mizar factores de riesgo. Asimismo, que familia y escuela aportan elementos formativos y de fortalecimiento personal, necesarios para que los jóvenes puedan afrontar sus propias dificultades cotidianas.

Con respecto a la herramienta del teatro para la convivencia, padres y profesores de las instituciones consideraron que es una excelente forma de trabajo que permitió no solo visibilizar las problemáticas, sino observar cómo mediante prácticas reflexivas se puede incidir en su transformación. Resaltaron que su aplicación en el contexto escolar posibilita evidenciar los roles que las diversas personas asumen frente a una problemática y cómo cuestionar dichos roles posibilita la búsqueda de alternativas de solución. También se reconoció que es una herramienta que convoca los intereses de los jóvenes, pues les brinda la posibilidad de intervenir de forma directa en aquellas situaciones de convivencia en sus contextos personales, familiares y sociales.

\section{Gestión intersectorial: foros y jornadas académicas en competencias ciudadanas y resolución de conflictos}

La formación en competencias ciudadanas y en el ejercicio de la resolución pacífica de conflictos se constituye en un desafío para los entes gubernamentales, para las instituciones educativas y para la sociedad colombiana en general, ya que se trata de un proceso que requiere pensar en la necesidad de realizar cambios en los niveles social y cultural que están más allá de la aplicación y apropiación de normas y políticas de convivencia ciudadanas.

Conscientes de estas realidades, en el proyecto Formación ciudadana: un reto para la convivencia pacífica en el departamento de Caldas, se estableció la realización de dos foros académicos en espacios comunitarios, estrategia que posibilitó poner el tema de la formación ciudadana y la resolución de conflictos en la escena de las discusiones y agendas de los gobiernos y las organizaciones locales. Los foros favorecieron aprendizajes colectivos a través de la participación de diversos actores de la comunidad en las ciudades de Chinchiná y Riosucio.

Los foros, además de una estrategia de socialización de información, se constituyeron en un ejercicio de análisis y de escucha de los discursos que circulan en dichas ciudades. En este sentido, la generación de este tipo de espacios fue valorada por los actores locales, como escenarios generadores de aprendizaje democráticos que favorecen las prácticas de autonomía y responsabilidad de las comunidades locales en la toma de decisiones y en el fortalecimiento de los vínculos familiares y comunitarios.

El desarrollo de los foros privilegió dinámicas de participación con el propósito de que los miembros de las organizaciones locales y la comunidad en 
general, visualizaran, desde sus propias realidades municipales, acciones que favorecieran la integración de los niños y jóvenes, a los procesos de sana convivencia en sus localidades. El foro en el municipio de Chinchiná estuvo centrado en el tema de la construcción de la democracia y la convivencia; por su parte, el foro de Riosucio, giró en torno a la formación ciudadana para la vivencia de una sexualidad sana, plena y responsable.

\subsection{Foro en el municipio de Chinchiná}

El foro tuvo por nombre: $1^{\text {er }}$ Foro Regional: Construyendo Democracia y Convivencia Ciudadana. Se desarrolló en el auditorio de la Institución Educativa Bartolomé Mitre y fue gestionado por la Secretaría de Educación de Caldas, la Red de Cultura Ciudadana y Democracia de Caldas y la Universidad Católica de Manizales. Su objetivo principal consistió en propiciar un espacio de interacción y reflexión para avanzar en la identificación y exploración de nuevos caminos y opciones de realización de propósitos comunes, para la consolidación de la red, de sus nodos y fortalecer las competencias ciudadanas desde la escuela y la sociedad en su conjunto, a fin de contribuir en la cultura política, la cultura de la legalidad y la cultura de la convivencia.

En el foro se llevaron a cabo tres ponencias: Cultura política y democracia, a cargo del señor Juan Pablo Castro Morales, director Nacional para la Democracia - Ministerio del Interior-; Convivencia ciudadana, ponencia del señor general Álvaro Pico Malaver, y la ponencia La escuela como escenario para la formación en competencias ciudadanas, del docente Silvio Cardona González, en representación de la Universidad Católica de Manizales. Al foro asistieron ciento cincuenta y nueve (159) personas entre las que se contaron estudiantes, docentes, miembros de entes territoriales, miembros de ONG, agentes comunitarios y miembros de la comunidad en general.

En síntesis, las conferencias y las intervenciones de los participantes dejaron entrever las acciones que en materia de competencias ciudadanas se vienen desarrollado a nivel nacional, regional y en particular, en las instituciones educativas, tanto en la dimensión formativa a todos los niveles, como en la dimensión de la participación ciudadana. Se resaltó, del mismo modo, la manera como los jóvenes, niños y niñas se reconocieron como ciudadanos dispuestos al ejercicio de sus deberes y conscientes de sus derechos.

Los participantes en el municipio de Chinchiná, reconocieron el foro como un evento constructivo; consideraron que aportó aspectos relevantes para el ejercicio de las actividades en el plano laboral, académico y personal. Entre las personas que respondieron el formulario de evaluación de la jornada se encontraban coordinadores de instituciones educativas de Chinchiná, estudiantes 
y actores de diversos sectores, quienes reconocieron el carácter dinámico y constructivo de la jornada.

\subsection{Foro en el municipio de Riosucio}

El Foro Formación ciudadana para la vivencia de una sexualidad sana, plena y responsable fue gestionado por la Secretaría de Educación de Caldas, el Instituto Colombino de Bienestar Familiar y la Universidad Católica de Manizales. En el foro participaron doscientas veintidós (222) personas entre las que se encontraban también estudiantes, docentes, miembros de entes territoriales, miembros de ONG, agentes comunitarios y miembros de la comunidad en general. Es importante resaltar el compromiso y apoyo de las autoridades y diversos organismos locales.

Entre los objetivos del foro estuvieron: reconocer cómo la formación ciudadana aporta significativamente a la sociedad para la vivencia de una sexualidad sana, plena y responsable; generar el debate y la conversación intersectorial sobre los factores de prevención y riesgos del embarazo en adolescentes y jóvenes del municipio y establecer compromisos por parte de los diferentes agentes participantes en el foro, que apoyen y permitan fortalecer los programas de prevención del embarazo en adolescentes y jóvenes en el municipio de Riosucio.

El foro comenzó con la ponencia: Multicausalidad del embarazo adolescente con enfoque diferencial, a cargo de Pilar Aguirre, psicóloga de la Universidad Javeriana, además de especialista en Logoterapia-Psicoterapia, Terapia Sexual y de Pareja, y Manejo del Duelo, y experta en educación sexual.

Luego de la conferencia central los participantes se distribuyeron en las cinco (5) mesas de trabajo previamente establecidas; en ellas se desarrollaron y abordaron las siguientes temáticas: mesa $n .^{\circ} 1$ : Acuerdo de voluntades; mesa n. ${ }^{\circ}$ 2: Fortalecimiento de la educación sexual; mesa n. ${ }^{\circ}$ 3: Territorialización del CONPES 147; mesa n. ${ }^{\circ}$ : Compromiso de los agentes educativos; mesa n. ${ }^{\circ}$ : La corresponsabilidad de la familia en la prevención del embarazo en adolescentes y jóvenes.

Luego, en plenaria general, se dio lectura al Acuerdo de Voluntades entre las distintas entidades y autoridades del municipio de Riosucio para la prevención del embarazo adolescente. Las reflexiones que se suscitaron y los compromisos asumidos resaltaron la importancia de la temática desarrollada y el manejo que esta requiere en los diferentes escenarios. Se observó un alto nivel de preocupación frente a la toma de conciencia acerca de las problemáticas de convivencia asociadas alrededor de los embarazos en niñas y adolescentes, y la necesidad de pensar en una educación sexual de forma integrada, así como las 
responsabilidades sociales de cada uno de los sectores que están directamente involucrados tanto en el nivel gubernamental, como en el institucional y familiar, sin dejar de reconocer las responsabilidades de los mismos jóvenes (Díaz, 2009).

En términos generales se establecieron compromisos puntuales para ampliar la Mesa departamental del CONPES a otros actores como Cultura, Deportes, Red Unidos y Juventud. Se habló del fortalecimiento de los SSAAJ (Servicios de Salud Amigable para Adolescentes y Jóvenes) mediante una delegación de personal para el funcionamiento de esta con énfasis en salud sexual, y de consolidar el portafolio de servicios para jóvenes y socializarlo en el ámbito municipal.

Se planteó, además, la necesidad de fortalecer el saber pedagógico en el marco del programa de educación sexual y la construcción de ciudadanía, a través de capacitación, seguimiento y desarrollo de acciones pedagógicas para la toma de decisiones activas, positivas y responsables (Escartin, 2003). Asimismo, se acordó el apoyo y fortalecimiento de relaciones intersectoriales y el compromiso de la SED y SEM para apoyar la capacitación de docentes y el desarrollo de proyectos institucionales que convoquen las acciones de las comunidades educativas.

La jornada culminó con un recorrido por los diversos stands que se organizaron para visibilizar las entidades, servicios e iniciativas que se desarrollan en la región con miras a promover sistemas de vida sana para niños y jóvenes. Entre las entidades que se hicieron presentes están: la SED, ICBF Centro Zonal Occidente, UCM, Hospital de Riosucio y la Secretaría de Integración Social del Departamento de Caldas.

El foro fue valorado de manera excelente por los participantes en el mismo. Entre sus percepciones se resaltaron la metodología empleada, los aportes conceptuales y la organización del evento; se valoraron, igualmente, el ambiente de trabajo y el clima de participación que se vivió a lo largo de la jornada. Al foro acudieron funcionarios de la alcaldía de Riosucio, rectores, coordinadores, docentes, estudiantes y padres de familia.

\section{Movilización social: cartilla}

El sexto componente del proyecto Formación Ciudadana: Un reto para la convivencia pacífica en el departamento de Caldas, constituía la elaboración y difusión de material gráfico - dos mil (2000) cartillas- con elementos referidos a la resolución pacífica de conflictos y a la vivencia de competencias ciudadanas, especialmente las competencias comunicativas y emocionales.

Acorde con los lineamientos formulados por la SED, la cartilla estuvo dirigida a los docentes de las instituciones educativas de Caldas como una herramienta 
pedagógica para la formación en ciudadanía. Dicha cartilla contó con el debido sustento teórico y conceptual, articulado a un lenguaje visual y recurriendo a elementos discursivos y paisajísticos propios de la cultura caldense. $\mathrm{El}$ apoyo teórico de la cartilla se fundamentó especialmente en la guía n. 49 del MEN.

La cartilla no fue concebida como la culminación de un proceso, sino como parte de la reflexión que ha de atravesar a los miembros de una sociedad, en proceso de consolidación de modos de convivencia más favorables para todos. La ciudadanía y su construcción conforman un movimiento conjunto, donde todos y cada uno de los miembros de una sociedad son responsables; en este sentido el quehacer de los educadores del sector público del departamento de Caldas tiene un papel decisivo.

El diseño de la cartilla no tuvo la intención de instruir sobre el tema específico de las competencias ciudadanas, sino más bien la de plasmar un contenido que resultara agradable a los sentidos, que suscitara pensamientos y propiciara el acercamiento de los lectores-educadores con su quehacer cotidiano, en su ejercicio docente, en su encuentro con el otro, niños-jóvenes-colegas, y también llegar al centro de la relación consigo mismo.

La cartilla, finalmente, hizo parte de los múltiples intentos que la sociedad colombiana realiza en el marco de la formación ciudadana como un interés colectivo y que específicamente se hace desde y en los espacios educativos, sin que por ello se considere que se trata de los más importantes. Resta reforzar la consciencia de que desde ellos -los espacios académicos-y desde los docentes como parte del engranaje educativo y social colombiano, mucho se puede hacer en la tentativa de ser ciudadanos más comprometidos con el devenir colectivo.

\section{Conclusiones}

La Universidad Católica de Manizales, como entidad operadora del proyecto de Formación ciudadana: un reto para la convivencia pacífica en el departamento de Caldas, entiende la formación en competencias ciudadanas como una dinámica de acercamiento que permite reconocer y potenciar los valores de las personas y de las comunidades. En este sentido, la formación en competencias ciudadanas, implicó un proceso respetuoso de articulación de los saberes formales y teóricos, con los conocimientos tradicionales que poseen las personas y las comunidades.

Las asesorías, talleres, cursos y demás actividades contempladas en el proyecto convocaron al equipo gestor de la Universidad Católica de Manizales, a desplegar actitudes de apertura y creatividad que favorecieran el reconocimiento de las situaciones de convivencia y promovieran, desde cada realidad particular, alternativas que contribuyeran a generar procesos de convivencia y solución pacífica de los conflictos cotidianos. 
Los diversos encuentros con los educadores y los directivos de los establecimientos educativos evidenciaron reconocimiento de las temáticas en torno a competencias ciudadanas. Los docentes tienen conocimiento del saber discursivo que promueven los diversos entes gubernamentales y frente al cual manifiestan diversidad de resistencias, actitud que dificulta cualquier proceso de articulación a sus propias vivencias y, por ende, a sus prácticas formativas cotidianas. Advertidos de esta circunstancia, la propuesta implementada por la Universidad Católica de Manizales planteó, en primera instancia, las realidades humanas de convivencia y cómo cada persona y cada comunidad pueden desplegar sus posibilidades en favor de la creación de condiciones de vida favorables para todos. Fue precisamente esta lógica la que posibilitó la creación de compromisos para hacer vívidas las competencias en sus comunidades educativas y para institucionalizarlas de manera más estructural.

El encuentro con los niños y jóvenes de las comunidades educativas de los establecimientos educativos de Caldas dejó entrever sus potencialidades y disposición para apropiarse de nuevos saberes y herramientas que les permitan mejorar sus interacciones con los compañeros y con la comunidad en general. Los chicos de los diversos municipios valoraron gratamente las posibilidades de participación y escucha de sus situaciones que el proyecto les posibilitó. En este sentido, establecieron correlaciones importantes entre ciertos estilos de vida y la ausencia de espacios, de oportunidades y capacidad de acogida de sus necesidades, manifestando reiteradamente que algunos educadores parecen no interesarles lo que acontece en las instituciones donde laboran.

Finalmente, cabe resaltar que la dimensión de un proyecto como este, en cuanto a cobertura y alcance, fue posible gracias a la articulación de varias instituciones de carácter público y privado, así como a la conjunción de saberes académicos, sociales e institucionales. Los aportes de cada una de las instancias comprometidas con el mismo fueron determinantes para el satisfactorio resultado obtenido.

\section{Referentes bibliográficos}

Álvarez, M. (2007). Sistematizar las prácticas, experiencias y proyectos. Gobernación de Antioquia; Universidad Luis Amigó: Medellín.

Boal, A. (2009). A Estética do Oprimido. Río de Janeiro: Garamond y Funarte MinC.

Chaux, E., Vargas, E., Ibarra, C. \& Minsk, M. (2013). Procedimiento básico para los establecimientos educativos. Documento final de la consultoría para la elaboración de la reglamentación de la Ley 1620 de 2013.

Chaux. Enrique y otros (2012). Competencias ciudadanas: de los estándares al aula. Una propuesta de integración a las áreas académicas.Universidad de los Andes. Bogotá. 
Díaz, C. (2009). Sexualidad. De la infancia a la adolescencia. Ser padres, ser madres hoy, volumen 3. Bogotá: Escuela de Estudios en Psicoanálisis y Cultura. Universidad Nacional.

Escartin, M., (2003). Abuso sexual a niños y niñas: preguntas y respuestas, una guía para acercarse al tema. Bogotá: D. C.: Asociación Afecto contra el Maltrato Infantil.

MEN. (2010). Guía pedagógica para la convivencia escolar. Guía n.2 28. Bogotá.

MEN. (2008). Guía para el mejoramiento institucional. De la autoevaluación al plan de mejoramiento. Guía n. 34. Bogotá.

MEN. (2008). Guías pedagógicas para la convivencia escolar. Ley 1620 de 2013-Decreto 1965 de 2013. Guía n. 49. Bogotá.

MEN. (2011). Orientaciones para la institucionalización de las competencias ciudadanas; Cartilla 1 Brújula. Bogotá.

MEN. (2011). Orientaciones para la institucionalización de las competencias ciudadanas; Cartilla 2 Mapa. Bogotá.

MEN. (2006). Estándares básicos de competencias en lenguaje, matemáticas, ciencias y ciudadanas. Bogotá.

Puerta Zapata, O. (1996). Evaluación y sistematización de proyectos sociales. Una metodología de investigación. Medellín: Universidad de Antioquia. Departamento de Trabajo Social.

Skliar, C \& Larrosa, J. Comp. (2009). Experiencia y alteridad en educación, 1 edición. Rosario: Homo Sapiens Ediciones.

Skliar, C. (2007). La educación (que es) del otro: argumentos y desiertos de argumentos pedagógicos, 1 ed. Buenos Aires: Ediciones Novedades Educativas. 
